\title{
Magnitud de la segregación escolar por nivel socioeconómico en España y sus Comunidades Autónomas y comparación con los países de la Unión Europea
}

\author{
Magnitude of school segregation by socioeconomic level in Spain \\ and its Autonomous Communities and comparison with the countries of the \\ European Union
}

\author{
F. Javier Murillo y Cynthia Martínez-Garrido'
}

\section{Resumen}

El objetivo de esta investigación es estimar la magnitud de la segregación escolar por nivel socioeconómico en España y sus Comunidades Autónomas, comparando los resultados con los países de la Unión Europea. Para ello se realiza una explotación especial de los datos PISA2015, utilizando los índices de Gorard y de Aislamiento. Los resultados indican que la segregación promedio en España en su dimensión de uniformidad es de 0,38 (índice de Gorard) y de 0,32 en su dimensión de exposición (índice de Aislamiento). Estos datos indican que España es uno de los países más segregados de Europa, con cifras análogas a los países del Este más segregados y muy alejadas de los países de su contexto más cercano. La segregación escolar en las Comunidades ofrece resultados extremos, algunas se encuentran entre las más bajas de Europa (Illes Balears, Galicia y Aragón) y otras como la Comunidad de Madrid con una segregación muy alta, solo superada por Hungría dentro de la Unión Europea. Con todo ello se observa la incidencia de las políticas educativas regionales respecto a los criterios de admisión de centros en la segregación escolar, mostrando que políticas como el fomento de la educación privada, de la competencia de centros mediante la publicación de rankings o la creación de un distrito único pueden configurar sistemas educativos inequitativos que atentan a la igualdad de oportunidades.

\section{Palabras clave}

Segregación escolar, nivel socioeconómico, España, Educación Secundaria, PISA.

\section{Abstract}

The aim of this research is to estimate the magnitude of school segregation by socioeconomic level in Spain and Autonomous Communities, comparing the results with the countries of the European Union. For this purpose, it performs a special exploitation of the PISA-2015 dataset, using the Gorard and Isolation indices. The results indicate that the average segregation is Spain in its unevenness dimension is 0.38 (Gorard index) and 0.32 in its exposure dimension (Isolation index). These findings indicate that Spain is one of the most segregated countries in Europe, similar to those countries in the East that are more segregated and far from the countries in their closest context. School segregation in the Autonomous Communities offers extreme results, some of which are among the lowest in Europe (Illes Balears, Galicia and Aragon) and others such as the Community of Madrid with a very high segregation, only surpassed by Hungary within the European Union. Our findings highlight the impact of regional educational policies on the admission criteria to schools into school segregation, showing that policies such as the promotion of private education, the competence of schools through the publication of rankings or the creation of a single district can set up unequal educational systems that attempts equal opportunities.

\section{Keywords}

School segregation, socioeconomic status, Spain, secondary education, PISA. 
Este articulo ha sido desarrollado en el marco del Proyecto $I+D+i$ de Excelencia «Escuelas en contextos socioeconómicamente desafiantes: Una aproximación desde la Educación para la Justicia Social de Investigación» Ref: EDU2014-56118-P. Financiado con fondos del Ministerio de Economía y Competitividad del Gobierno de España.

\section{Introducción}

La escuela juega un papel determinante en la equidad y cohesión social. Cuando se ve limitada en su papel de educar a través de la convivencia de personas con diferentes características personales o sociales se convierte en un factor de desigualdad, de tal forma que no solo reproduce las desigualdades, las legitima y potencia. La segregación escolar, fenómeno por el cual los estudiantes se distribuyen desigualmente en las escuelas en función de alguna de sus características es, por tanto, uno de los factores que contribuyen en mayor medida a impedir una verdadera igualdad de oportunidades y generar desigualdad social. Su existencia, bien sea por la situación socioeconómica de las familias, bien sea por el país de nacimiento del estudiante, por su pertenencia a un grupo étnico-cultural o, incluso, por su rendimiento académico previo, contribuye a la generación de fenómenos de exclusión y desintegración social.

Así, existe una fructífera línea de investigación centrada en la segregación escolar como indicador de equidad educativa. De esta manera, se ha desarrollado un importante número de trabajos que dan cuenta de su magnitud y evolución en muchos países, así como de sus causas y efectos en los estudiantes y en la sociedad (por ejemplo, Coleman et al., 1975; Cheng y Gorard, 2010; Dupriez, 2010; Dupriez y Vandenberghe, 2004; Goodman, 1972; Gorard y Hordsoy, 2013; Jenkins et al., 2008; Katz, 1964; Murillo y Martínez-Garrido, 2017a; Orfield y Eaton, 1997; Reardon y Owens, 2014; Stephan, 2013).

Sin embargo, son aun pocos los estudios que aportan información sobre la segregación escolar en España. Es posible que la no disponibilidad de datos representativos para el conjunto del Estado y el escaso interés por profundizar en las inequidades del sistema educativo estén detrás de ello. Los escasos trabajos desarrollados han estudiado mayoritariamente la segregación escolar por origen nacional en algunas regiones o localidades (Benito y González-Balletbó, 2007; Ferrer et al., 2008; Mancebón-Torrubia y Pérez-Ximénez de Embún, 2005; Sánchez Hugalde, 2007; Síndic, 2008). Aunque también destacan algunos trabajos que abordan la segregación escolar por origen nacional para el conjunto del Estado español (Benito et al., 2014; Mancebón-Torrubia y Pérez-Ximénez de Embún, 2009; Murillo et al., 2017b) e investigaciones que estiman la segregación escolar en función del nivel socioeconómico y cultural de las familias de los estudiantes (Ferrer et al., 2008; Mancebón-Torrubia y Pérez-Ximénez de Embún, 2010).

En esta investigación se busca estimar la magnitud de la segregación escolar por nivel socioeconómico en el conjunto del Estado español y en cada Comunidad Autónoma, contextualizándolas con la situación de países de su entorno. Para ello, se hace una explotación especial de los datos del Programa Internacional para la Evaluación de Alumnos (PISA) correspondientes a 2015 (OCDE, 2016), últimos disponibles hasta el momento.

\section{Revisión de la literatura}

Existe consenso en considerar la sentencia judicial del caso «Brown v. Board of Education», de 17 de mayo de 1954, como el revulsivo que dio inicio al interés del estudio de la segregación escolar (Reardon y Owens, 2014). En ella, la Corte Suprema de los Estados Unidos declaró ilegal la segregación de los 
estudiantes en distintas escuelas en función del color de su piel. Con ello, en los años 60 y 70 se multiplicaron los trabajos en Estados Unidos que buscaban estimar la magnitud de la segregación étnico-racial, así como identificar sus causas y sus efectos sobre el rendimiento (Coleman et al., 1975; Goodman, 1972; Katz, 1964; Orfield y Eaton, 1997). Sin embargo, habría que esperar a los años 80 para que la investigación se preocupara por el estudio de la segregación escolar por nivel socioeconómico. Y quizá sea el estudio de Wilson (1987), titulado «The Truly Disadvantaged», en el que se describen las desventajas de la concentración de familias de clases sociales más desfavorecidas en algunas escuelas, uno de los pioneros.

En estos años ha disminuido el número de trabajos que abordan la segregación escolar étnico-racial, probablemente debido a que las evidencias empíricas muestran que este tipo de segregación es cada vez menor. Stroub y Richards (2013), por ejemplo, afirman que ahora es un $10 \%$ menor que hace una década. Sin embargo, la investigación sobre segregación escolar por origen nacional (Frankenberg et al., 2003; Karsten, 2010; Murillo y Martínez-Garrido, 2017a; Orfield et al., 2014), así como por nivel socioeconómico (Cheng y Gorard, 2010; Dupriez, 2010; Jenkins et al., 2008; Reardon y Owens, 2014; Stephan, 2013) están aumentando en número y calidad en todo el mundo.

En lo que respecta a la segregación escolar por nivel socioeconómico, recientes trabajos han aportado evidencias empíricas de su magnitud en diferentes países. En Estados Unidos, por ejemplo, Reardon y Owens (2014) señalan que la segregación escolar en las escuelas públicas de Educación Primaria es de 0,11 (valor medido con el índice de Raíz Cuadrada). En Inglaterra, Gorard y Hordsoy (2013) utilizan los datos del censo escolar con la información del 93\% de los estudiantes del país y sus resultados muestran que el valor de la segregación escolar por nivel socioeconómico es de 0,33 tanto en las escuelas de etapa Primaria como Secundaria (valor medido a través del índice de Gorard). Según los autores, estos valores pueden variar hasta en 1,6 décimas de una zona a otra del país, siendo el norte de Inglaterra donde se encuentran las escuelas con menor segregación socioeconómica (0,25 puntos). De acuerdo con Dupriez y Vandenberghe (2004), Bélgica es uno de los países europeos donde las escuelas están más segregadas por nivel socioeconómico, 0,48 en la zona francófona del país y 0,41 en la zona flamenca (valores medidos a través del índice de Disimilitud). Alemania también parece ser uno de los países más segregados, según Jenkins y colaboradores (2008) la segregación escolar por nivel socioeconómico es 0,41 medido según el índice de Disimilitud, su valor calculado con el índice de Raíz Cuadrada es 0,13.

Otros trabajos utilizan los datos de las evaluaciones internacionales como PISA o TERCE para mostrar una imagen comparativa de la segregación escolar por nivel socioeconómico en diferentes países de la OCDE o de América Latina. Entre ellos, destaca la investigación de Ferrer y colaboradores (2008) en la que se estima la magnitud de la segregación escolar por nivel socioeconómico en 27 países haciendo una explotación especial de los datos PISA-2006 y estimando el índice de Disimilitud para el 25\% de los estudiantes con familias de menor nivel socioeconómico (Q1). Los resultados apuntan a que Finlandia y Noruega son los países con menor segregación $(0,28)$, mientras que Hungría $(0,54)$, Bélgica, Francia $(0,44)$, Alemania y Grecia $(0,43)$ son los países con mayor índice de segregación, ocupando España $(0,40)$ el décimo puesto de entre los más segregados. También es interesante el trabajo de Vázquez (2012), en el que se encontró, usando datos de PISA-2009 y mediante el índice de Disimilitud con la mediana de estudiantes como grupo minoritario, que los países escandinavos son los que menores índices de segregación escolar por nivel socioeconómico tienen de todo el mundo, entre 0,26 y 0,31. En el extremo opuesto, Perú, Chile y Tailandia, con más de 0,5 puntos, son los países más segregados. España ocupa una posición intermedia, con un índice de segregación escolar por nivel socioeconómico de 0,39. 
Más reciente es el trabajo de Murillo y Martínez-Garrido (2017a) que usa los datos del Tercer Estudio Comparativo y Explicativo (TERCE) de la UNESCO para estimar la magnitud de la segregación por nivel socioeconómico en las escuelas de Educación Primaria en 17 países de América Latina. Los resultados muestran cifras más altas a las mencionadas en los países europeos; concretamente un valor de 0,54 promedio para el 25\% de los estudiantes de familias con menor nivel socioeconómico de la Región (también con el índice de Disimilitud). Los autores destacan las grandes diferencias entre países; efectivamente, República Dominicana y Uruguay son los países de América Latina con una menor segregación escolar, mientras que Honduras, México y Perú, son los países con mayor segregación. Resultados que son coherentes a los aportados por Vázquez (2012) en Educación Secundaria y que muestran un preocupante alto nivel de segregación escolar por nivel socioeconómico en la Región.

El estudio de la segregación escolar en España tiene apenas una década de existencia y aún se han desarrollado pocos trabajos. Entre ellos es posible encontrar investigaciones que abordan la segregación escolar por origen nacional del alumnado y otras que estudian la segregación escolar por nivel socioeconómico.

Entre los estudios que han abordado la segregación por origen nacional de los estudiantes destacan los trabajos realizados por Benito y González-Balletbó (2007) y Sánchez Hugalde (2007), junto con los informes elaborados por instituciones como la Fundació Jaume Bofill (Bonal, 2012; Ferrer et al., 2011) o el Síndic de Grenges de Cataluña (Síndic, 2008), todos ellos centrados en Cataluña. Así, Sánchez Hugalde (2007) estudia el nivel de segregación escolar por origen nacional en los municipios con más de 20.000 habitantes con datos del curso 2002/03. Según la autora, el nivel más alto de segregación se obtiene en Barcelona $(0,62)$ y el menor en el Valle de Arán $(0,19)$ (valores medidos a través del índice de Disimilitud). Por su parte, Benito y González-Balletbó (2007) estiman el grado de segregación escolar por origen nacional en 12 municipios de Cataluña; sus resultados muestran que el nivel de segregación escolar es de 0,4 , medido a través del índice de Gorard.

El informe elaborado por el Sindic de Greuges de Catalunya (Síndic, 2008) estudia la evolución de la magnitud de la segregación escolar por origen nacional en centros docentes de Educación Primaria y de Educación Secundaria en Cataluña desde finales de los años 90 y hasta 2006. Los resultados muestran tres grandes etapas en la magnitud de la segregación escolar por origen nacional en Cataluña: (i) finales de la década de los años 90, en la que había poca intensidad de flujos migratorios y los niveles de segregación eran valores altos (0,55 en Primaria, y 0,47 en Secundaria); (ii) del año 2000 a 2004, cuando hubo una etapa de decrecimiento del nivel de segregación (0,45 en Primaria y 0,37 en Secundaria); y (iii) durante los años 2005 y 2006, en los que los niveles de segregación escolar se mantienen constantes alcanzando valores moderados (0,46 en Primaria y 0,35 en Secundaria) (valores medidos a través del índice de Disimilitud).

También son escasos los trabajos que analizan la segregación escolar por nivel socioeconómico en España (Benito et al., 2014; Fernández-Enguita, 2008; Mancebón-Torrubia y Pérez-Ximénez de Embún, 2008, 2010). Por ejemplo, el trabajo de Mancebón-Torrubia y Pérez-Ximénez de Embún (2010) determina la segregación escolar por nivel socioeconómico del conjunto de España utilizando datos de PISA-2006. De acuerdo con los autores, la segregación promedio para el conjunto del Estado español es de 0,39, estimado mediante índice de Disimilitud. Por su parte, la investigación de Benito y colaboradores (2014) analiza el efecto de la segregación escolar por nivel socioeconómico sobre el rendimiento 
académico de los estudiantes en Lectura utilizando en PISA-2009. Los resultados indican que España, junto con Islandia, son los dos únicos países del estudio donde no se encuentran efectos estadísticamente significativos del nivel de segregación sobre los resultados académicos obtenidos por los estudiantes.

Como se ha visto, la investigación en España sobre segregación escolar por nivel socioeconómico está prácticamente sin abordar. Los pocos trabajos previos realizados no nos permiten obtener una visión global del estudio de la segregación escolar en el país. Es por ello que en esta investigación se estima la magnitud de la segregación escolar de carácter socioeconómica en el conjunto de España y en cada Comunidad Autónoma y se contextualiza con los datos de los países de la Unión Europea.

\section{Medición de la Segregación escolar}

La anterior revisión evidencia una falta de acuerdo entre los investigadores en torno al índice de segregación a utilizar. Pero no es un fenómeno nuevo, hace ya 30 años Peach (1975) habló de una «guerra de índices» para estimar la segregación. Esta misma idea fue utilizada posteriormente por Taylor y colaboradores (2000), Gorard y Taylor (2002), Allen y Vignoles (2007) o Murillo (2016) y dan muestra de la falta de consenso tanto en el propio concepto de segregación hasta de las bondades de un índice frente al resto.

Es posible distinguir dos dimensiones en la conceptualización de la segregación escolar: la dimensión de uniformidad y la dimensión de exposición. Según la dimensión de uniformidad se define la segregación escolar como la distribución desigual de los estudiantes en las escuelas en función de sus características personales o sociales (por ejemplo, Dupriez, 2010; Frankel y Volij, 2011; Ireson y Hallam, 2001). Según la dimensión de exposición, se entiende por segregación escolar la probabilidad de que un estudiante de un grupo minoritario se encuentre en la escuela con otro de su mismo grupo (Allen y Vignoles, 2007; Johnston et al., 2004).

Para estimar la segregación escolar desde la perspectiva de la uniformidad, los índices más utilizados son el índice de Disimilitud (Duncan y Duncan, 1955), el índice de Gorard (1998), el índice de la Raíz Cuadrada (Hutchens, 2004), el índice de Brecha por Centiles (Watson, 2009), o el índice Inclusión socioeconómica (Murillo, 2016). Para medir la segregación como exposición, el índice más habitual es el de Aislamiento (Lieberson, 1981).

La presente investigación busca aportar una visión global del fenómeno de la segregación escolar por nivel socioeconómico en España, por eso, se aborda su interpretación desde las dos dimensiones: uniformidad y exposición. Para ello, en primer lugar, se utiliza el índice de Gorard (G) que se define como «la desviación proporcional de los estudiantes del grupo minoritario con respecto a una completa distribución de la pobreza» (Gorard, 2006: 3). En segundo término, se utiliza el índice de Aislamiento (A) que, se estima como la probabilidad de que un miembro del grupo minoritario se encuentre en su escuela con otro miembro de su mismo grupo. De esta forma, cuanto mayor sea el aislamiento del grupo, menor será su exposición a los miembros del resto de grupos. De acuerdo con Echenique y Fryer (2007), el índice de Aislamiento está fuertemente influido por la participación relativa del grupo minoritario en la población total de estudiantes dado que capta el grado de contacto potencial de los estudiantes de este grupo.

La naturaleza de la variable por la que se define la segregación escolar (de escala o nominal) es clave para identificar el grupo minoritario. En aquellos casos en los que se estudie la segregación escolar de una variable nominal (origen nacional, étnico-cultural...), el grupo minoritario es fácilmente identificable. Sin embargo, si la variable criterio es de escala (nivel socioeconómico, rendimiento académico previo...), es necesario decidir los puntos de corte que definen el grupo minoritario. Así, se han utilizado la mediana 
(Vázquez, 2012), el primer cuartil (Ferrer et al., 2008), el percentil 30 (Orfield, 2009), el primer quintil (Andersson y Kährik, 2015) o el primer cuartil, el cuarto y su promedio (Murillo, 2016).

Para estimar el índice de Gorard y el de Aislamiento en esta investigación se ha optado por cuatro consideraciones de grupo minoritario: el percentil 10, el cuartil 1, el cuartil 3 y el percentil 90; es decir el $10 \%$ y el $25 \%$ de los estudiantes con familias de menor y de mayor nivel socioeconómico y cultural. El resultado promedio de los cuatro permite obtener una imagen global del nivel de segregación por nivel socioeconómico.

\section{Método}

La presente investigación busca estimar la magnitud de la segregación escolar por nivel socioeconómico en el conjunto del Estado español y en cada Comunidad Autónoma, contextualizándolas con la situación de los países de la Unión Europea. Para dar respuesta a este objetivo se hace una explotación secundaria de los datos del Programa Internacional para la Evaluación de Alumnos (PISA) de 2015 (OCDE, 2016). El propósito de PISA es evaluar cada tres años el nivel de adquisición de conocimientos de los estudiantes de 15 años en tres áreas temáticas clave: Lectura, Matemáticas y Ciencias. Cada una de las evaluaciones de PISA profundiza en un área concreta: Lectura (años 2000 y 2009), Matemáticas (años 2003 y 2012), y Ciencias (años 2006 y 2015). Igualmente, el programa recoge una amplia gama de resultados educativos, entre los que se encuentran: la motivación de los alumnos por aprender, la concepción que éstos tienen sobre sí mismos y sus estrategias de aprendizaje; así como información de factores asociados al aprendizaje que permite contextualizar y, hasta cierto punto, explicar los resultados obtenidos

En la edición de PISA-2015 participaron en total cerca de 540.000 estudiantes, representando alrededor de 29 millones de jóvenes de 15 años escolarizados en los 72 países y economías participantes (OCDE, 2016). Para este estudio se seleccionó, en primer lugar, los datos correspondientes a España. La muestra española está compuesta por 39.066 estudiantes, de 1.177 escuelas. De ellos, la muestra para el total de Estado español es de 6.736 estudiantes y 201 centros, y el resto de cada una de las 17 Comunidades Autónomas, con un tamaño promedio de 1.800 estudiantes y 54 centros por Comunidad (excepto el País Vasco que duplicó la muestra). En segundo lugar, se analizaron los datos de 27 de los 28 países de la Unión Europea, la excepción fue Chipre que no participó en PISA-2015. En este caso, la muestra en cada país fue de 6.531 estudiantes y 245 centros educativos de promedio. Con todo ello, la muestra total para este estudio es de 208.873 estudiantes y 7.553 centros. Su distribución detallada se encuentra en la tabla I.

PISA utiliza un muestreo estratificado en dos etapas. Las unidades de muestreo de la primera etapa son las escuelas con estudiantes de 15 años de edad. Las escuelas se seleccionan sistemáticamente a partir de una lista nacional completa de todas las escuelas elegibles para PISA, con probabilidad de selección proporcional al número estimado de estudiantes de 15 años matriculados en dicha escuela. Antes del muestreo, las escuelas elegibles en cada país se asignan a grupos mutuamente excluyentes basados en características escolares explícitas. Para el caso español, las variables de estratificación explícita son: titularidad del centro, Comunidad Autónoma, tamaño de la escuela y modalidad de enseñanza en el País Vasco. Las unidades de muestreo en la segunda etapa son los estudiantes de 15 años de las escuelas incluidas en la muestra. Para cada país se estableció un tamaño de clúster objetivo (35 estudiantes, aunque algunos países han optado por valores alternativos). Para aquellas escuelas donde hay más de 35 estudiantes con 15 años se seleccionó a 35 de ellos con igual probabilidad y para aquellas escuelas con menos de 35 estudiantes, se seleccionaron todos los estudiantes (OCDE, 2015). 
Tabla I. Muestra del estudio

\begin{tabular}{lcc} 
& N. ${ }^{\circ}$ ESTUDIANTES & N. ${ }^{\circ}$ ESCUELAS \\
\hline ESPAÑA & 6.736 & 201 \\
\hline Andalucía & 1.813 & 54 \\
\hline Aragón & 1.798 & 53 \\
\hline Ppdo. de Asturias & 1.790 & 54 \\
\hline Illes Balears & 1.797 & 54 \\
\hline Canarias & 1.842 & 54 \\
\hline Cantabria & 1.924 & 56 \\
\hline Castilla y León & 1.858 & 57 \\
\hline Castilla - La Mancha & 1.889 & 55 \\
\hline Caraluña & 1.769 & 52 \\
\hline Extremadura & 1.809 & 53 \\
\hline Galicia & 1.865 & 59 \\
\hline La Rioja & 1.461 & 47 \\
\hline Com. de Madrid & 1.808 & 51 \\
\hline Región de Murcia & 1.796 & 53 \\
\hline C. Foral de Navarra & 1.874 & 52 \\
\hline País Vasco & 3.612 & 119 \\
\hline Comunitat Valenciana & 1.625 & 53 \\
\hline & & 53 \\
\hline
\end{tabular}

\begin{tabular}{|c|c|c|}
\hline & N..$^{\circ}$ ESTUDIANTES & N. ${ }^{\circ}$ ESCUELAS \\
\hline ALEMANIA & 6.504 & 256 \\
\hline AUSTRIA & 7.007 & 269 \\
\hline BÉLGICA & 9.651 & 288 \\
\hline BULGARIA & 5.928 & 180 \\
\hline CROACIA & 5.809 & 160 \\
\hline DINAMARCA & 7.161 & 333 \\
\hline ESLOVAQUIA & 6.350 & 290 \\
\hline ESLOVENIA & 6.406 & 333 \\
\hline ESTONIA & 5.587 & 206 \\
\hline FINLANDIA & 5.882 & 168 \\
\hline FRANCIA & 6.108 & 252 \\
\hline GRECIA & 5.532 & 211 \\
\hline HUNGRÍA & 5.658 & 245 \\
\hline IRLANDA & 5.741 & 167 \\
\hline ITALIA & 11.583 & 474 \\
\hline LETONIA & 4.869 & 250 \\
\hline LITUANIA & 6.525 & 311 \\
\hline LUXEMBURGO & 5.299 & 44 \\
\hline MALTA & 3.634 & 59 \\
\hline PAÍSES BAJOS & 5.385 & 187 \\
\hline POLONIA & 4.478 & 169 \\
\hline PORTUGAL & 7.325 & 246 \\
\hline REINO UNIDO & 14.157 & 550 \\
\hline REP. CHECA & 6.894 & 344 \\
\hline RUMANÍA & 4.876 & 182 \\
\hline SUECIA & 5.458 & 202 \\
\hline
\end{tabular}

Total muestra

208.873

7.553

Fuente: elaboración propia a partir de datos de PISA-2015.

Como variable criterio se ha utilizado el constructo estimado por PISA «índice de la situación económica, social y cultural de la familia estudiante» (ESCS). Se trata de una variable tipificada que recoge la información de las siguientes variables: índice socioeconómico internacional de situación laboral (ISEI), nivel educativo más alto de los padres del estudiante, índice de la riqueza de la familia, índice de recursos educativos del hogar, e índice de posesiones relacionadas con la cultura en el domicilio familiar.

Como se ha señalado, para estimar la segregación escolar de carácter socioeconómico se utilizan dos índices de información complementarios: el índice de Gorard y el de Aislamiento. Por una parte, el índice de Gorard estudia la proporción que representa a los estudiantes del grupo minoritario en una escuela con respecto a cómo ese grupo minoritario se encuentra representado en un área geográfica concreta (ya sea a nivel de comunidad autónoma, país, distrito...), y se interpreta como la tasa de estudiantes que ten- 
drían que cambiar de escuela para lograr su distribución igualitaria dentro del área geográfica de análisis, para que la segregación sea 0 . Se ha optado por este índice por ser el más común entre los investigadores.

En segundo término, se usa el llamado índice de Aislamiento (A). El mismo estima la probabilidad de que un miembro del grupo minoritario se encuentre en su escuela con otro miembro de su mismo grupo. Este índice permite comprender la dimensión de exposición de la segregación escolar.

Matemáticamente se expresan de la siguiente forma:

$$
G=\frac{1}{2} \sum_{i=1}^{k}\left|\frac{x_{1 i}}{X_{1}}-\frac{T_{i}}{T}\right| \quad A=\sum_{i=1}^{k} \frac{x_{1 i}}{X_{1}} \frac{x_{1 i}}{T_{i}}
$$

Donde para cada Comunidad Autónoma o país,

x1i es el número de estudiantes del grupo minoritario en la escuela i.

X1 es el número total de estudiantes minoritarios en todas las escuelas de la Comunidad Autónoma o país.

Ti es número total de alumnos en la escuela i.

T es el número total de alumnos en la Comunidad Autónoma o país.

Para calcular tanto el índice de Gorard como el de Aislamiento se han considerado cuatro criterios de elección de grupo minoritario:

- El 10\% de los estudiantes con familias de menos nivel socioeconómico y cultural (P10).

- El 25\% de los estudiantes con familias de menos nivel socioeconómico y cultural (Q1).

- El 25\% de los estudiantes con familias de mayor nivel socio-económico y cultural (Q4).

- El 10\% de los estudiantes con familias de mayor nivel socioeconómico y cultural (P90).

Y para dar una imagen global más fácilmente comparable, se estima el promedio de las cuatro estimaciones para ambos índices. Para el cálculo de ambos índices se ha tenido en cuenta los pesos finales de cada estudiante, que suministra la propia base de datos PISA-2015 para cada escuela.

\section{Resultados}

El índice de segregación escolar de carácter socioeconómico promedio del conjunto de Estado español en su dimensión de uniformidad es de 0,38 (medido a través del índice de Gorard) y en su dimensión de exposición es de 0,32 (estimado mediante el índice de Aislamiento).

Si se analiza la segregación para cada grupo minoritario (gráfico 1) se observan dos detalles relevantes. En primer lugar, destaca el hecho de que la magnitud de la segregación varía en función del tamaño del grupo minoritario considerado, pero con un efecto diferente para cada uno de los dos índices utilizados. Para el índice de Gorard (dimensión de uniformidad), es mayor cuanto más pequeño sea el grupo minoritario, de tal forma que es más alta para el P10 y para el P90 (que suponen en 10\% del total), que para el Q1 y Q4 (que suponen un 25\%); mientras que para el índice de Aislamiento (dimensión de exposición) se produce el fenómeno contrario. En todo caso, son características de los índices que, por sí solas, explican poco de la situación española. 
Más interesante es comprobar que la segregación es más alta para el Q4 que para el Q1 y para el P90 que para el P10, y esto sucede en ambos índices. Este hecho que, como luego veremos, no se da en todos los países, pero si en todas las Comunidades Autónomas españolas, indica que la segregación para los estudiantes con familias con mayor nivel socioeconómico es mayor que para las de menor. Lo que habla de una tendencia a la segregación «por arriba» del sistema educativo español, es decir, a la «elitización» del mismo.

Gráfico 1. Magnitud de la segregación escolar en España. Índice de Gorard e índice de Aislamiento para el $10 \%$ de los estudiantes con familias de menos nivel socioeconómico y cultural (P10), para el $25 \%$ de los estudiantes con familias de menos nivel socioeconómico y cultural (Q1), $25 \%$ de los estudiantes con familias de mayor nivel socio-económico y cultural (Q4)), y para el $10 \%$ de los estudiantes con familias de mayor nivel socioeconómico y cultural (P90)
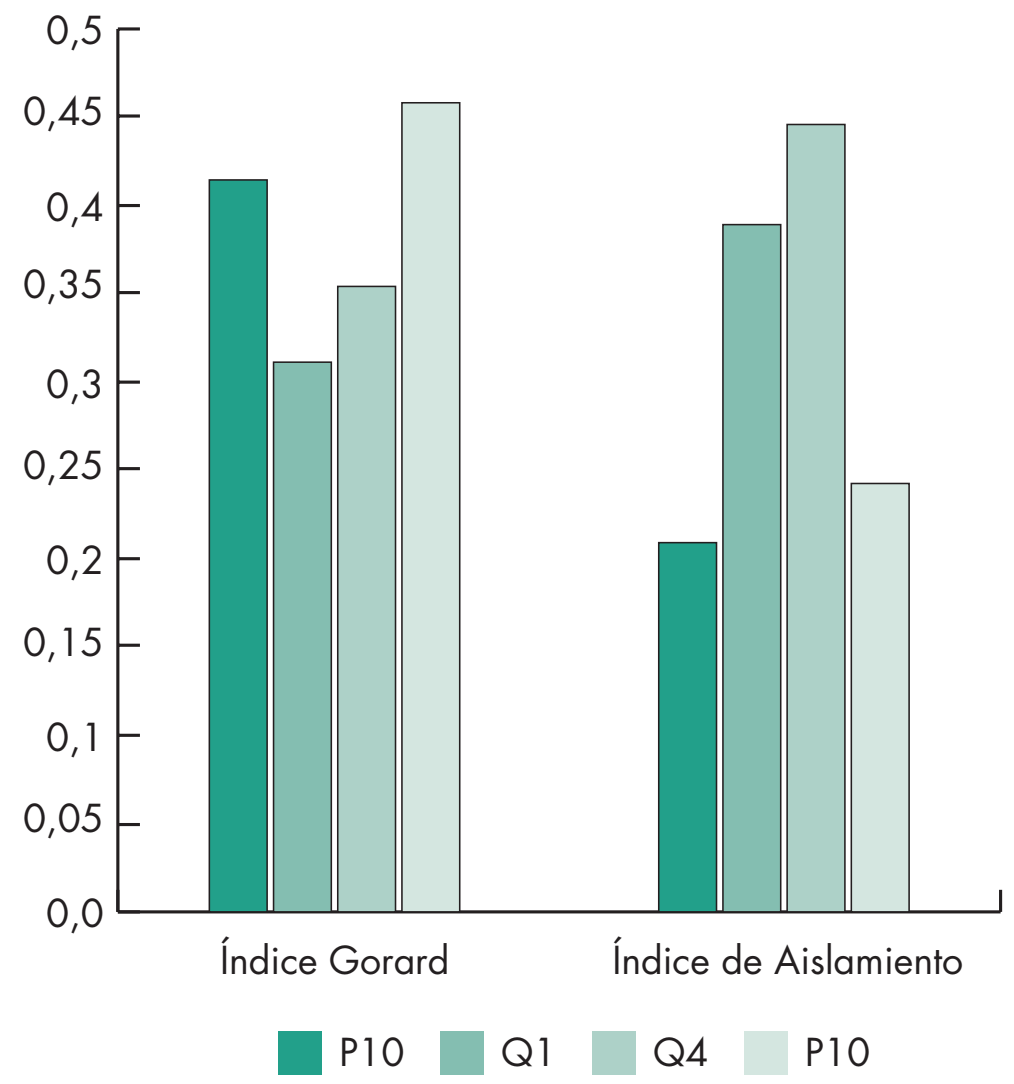

Fuente: elaboración propia con datos de PISA-2015.

Estas cifras, sin embargo, dicen poco si no se ponen en el contexto de lo que acontece en los países de su entorno.

\subsection{Segregación escolar en España en comparación con los países de su entorno}

Es posible afirmar que España se encuentra entre los países de la Unión Europea con un sistema educativo en Educación Secundaria más segregado (gráfico 2). Efectivamente, la magnitud de su segregación en su dimensión de uniformidad es solo superada por países del Este tales como Bulgaria $(0,39)$, Eslovaquia $(0,40)$, República Checa $(0,40)$, Rumania $(0,41)$ y Hungría $(0,46)$; y alejado de países de su entorno como Francia $(0,37)$, Alemania $(0,37)$, Italia $(0,36)$ o Portugal $(0,36)$. 


\section{Gráfico 2. Segregación escolar por nivel socioeconómica en países de la Unión Europea. Índice de Gorard promedio}

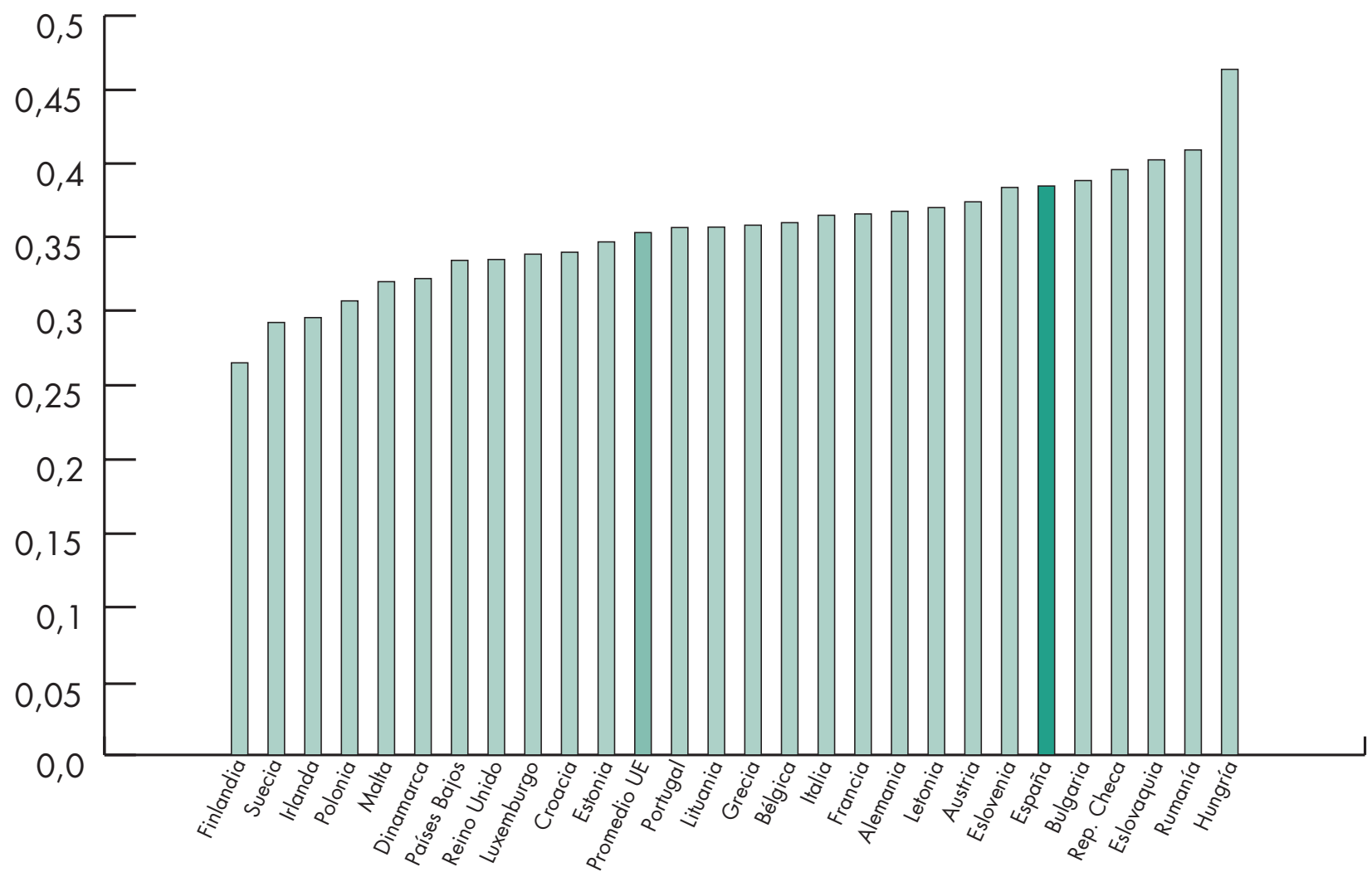

Fuente: elaboración propia a partir de datos de PISA-2015.

Más interesante resulta el análisis de la magnitud de la segregación para cada uno de los grupos minoritarios considerados (tabla II). Así:

- Para el 10\% de los estudiantes de familias de menor nivel socioeconómico, se observa que España $(0,41)$ se encuentra en una situación intermedia en la Unión Europea, muy cerca del promedio $(0,41)$ y más baja que países como Francia $(0,43)$, Alemania $(0,43)$ o Bélgica $(0,42)$.

- Para el 25\% de los estudiantes con familias de menor nivel socioeconómico, España $(0,31)$ se sitúa como el sexto país con mayor segregación, solo superado por países del Este europeo, y lejos de los países de su entorno más cercano.

- Para el 25\% de los estudiantes con familias de mayor nivel (Q4), España es el tercer país más segregado de toda la Unión Europea $(0,35)$, solo superado por Rumania $(0,35)$ y Hungría $(0,39)$.

- Para el 10\% superior, de nuevo España se vuelve a situar entre los más segregados $(0,46)$, en este caso el cuarto más segregado de Europa, solo por detrás de Republica Checa, Hungría y Rumania.

Estos datos nos aportan una imagen de la gran segregación existente en España para todos los colectivos. Únicamente, para el 10\% de los estudiantes con familias de menor nivel socioeconómico, su situación está en el promedio europeo. 


\section{Tabla II. Segregación escolar por nivel socioeconómico en España y su comparación con los países de la Unión Europea. Índice de Gorard para P1, Q1, Q4 y P90 como grupos minoritarios, y promedio}

\begin{tabular}{|c|c|c|c|c|c|}
\hline & P10 & Q1 & Q4 & P90 & PROMEDIO \\
\hline ESPAÑA & 0,4145 & 0,3113 & 0,3542 & 0,4582 & 0,3846 \\
\hline ALEMANIA & 0,4252 & 0,2959 & 0,3109 & 0,4389 & 0,3677 \\
\hline AUSTRIA & 0,4168 & 0,2865 & 0,3396 & 0,4535 & 0,3741 \\
\hline BÉLGICA & 0,4196 & 0,3061 & 0,3147 & 0,3998 & 0,3600 \\
\hline BULGARIA & 0,4885 & 0,3374 & 0,3197 & 0,4082 & 0,3885 \\
\hline CROACIA & 0,3728 & 0,2401 & 0,3040 & 0,4430 & 0,3400 \\
\hline DINAMARCA & 0,3891 & 0,2581 & 0,2593 & 0,3824 & 0,3222 \\
\hline ESLOVAQUIA & 0,5251 & 0,3152 & 0,3185 & 0,4511 & 0,4025 \\
\hline ESLOVENIA & 0,4447 & 0,3007 & 0,3398 & 0,4501 & 0,3838 \\
\hline ESTONIA & 0,4016 & 0,2927 & 0,2752 & 0,4187 & 0,3470 \\
\hline FINLANDIA & 0,2962 & 0,2295 & 0,2379 & 0,2972 & 0,2652 \\
\hline FRANCIA & 0,4324 & 0,2999 & 0,3307 & 0,4008 & 0,3659 \\
\hline GRECIA & 0,4245 & 0,2952 & 0,3040 & 0,4096 & 0,3583 \\
\hline HUNGRÍA & 0,5853 & 0,3870 & 0,3858 & 0,4968 & 0,4637 \\
\hline IRLANDA & 0,3314 & 0,2375 & 0,2552 & 0,3591 & 0,2958 \\
\hline ITALIA & 0,4377 & 0,3000 & 0,2930 & 0,4291 & 0,3650 \\
\hline LETONIA & 0,4491 & 0,2982 & 0,3008 & 0,4327 & 0,3702 \\
\hline LITUANIA & 0,4438 & 0,3137 & 0,3004 & 0,3699 & 0,3570 \\
\hline LUXEMBURGO & 0,3221 & 0,2827 & 0,3432 & 0,4068 & 0,3387 \\
\hline MALTA & 0,3383 & 0,2701 & 0,2786 & 0,3934 & 0,3201 \\
\hline PAÍSES BAJOS & 0,3652 & 0,2623 & 0,2979 & 0,4127 & 0,3345 \\
\hline POLONIA & 0,3540 & 0,2564 & 0,2504 & 0,3678 & 0,3071 \\
\hline PORTUGAL & 0,3865 & 0,2884 & 0,3205 & 0,4313 & 0,3567 \\
\hline REINO UNIDO & 0,3583 & 0,2638 & 0,2952 & 0,4232 & 0,3351 \\
\hline REP. CHECA & 0,4509 & 0,3032 & 0,3393 & 0,4900 & 0,3959 \\
\hline RUMANÍA & 0,4395 & 0,3179 & 0,3567 & 0,5227 & 0,4092 \\
\hline SUECIA & 0,3376 & 0,2306 & 0,2572 & 0,3444 & 0,2925 \\
\hline Promedio Unión Europea & 0,4067 & 0,2865 & 0,3049 & 0,4151 & 0,3533 \\
\hline
\end{tabular}

Fuente: elaboración propia con datos de PISA-2015.

Considerando la dimensión de exposición los resultados son muy parecidos, España se encuentra en una posición a) un poco por debajo del promedio de la Unión Europea en P10, b) el sexto país en Q1, c) el tercero en Q4, y d) el cuarto en P90. Cifras, sin duda, preocupantes (tabla III). 
Tabla III. Segregación escolar por nivel socioeconómico en España y su comparación con diferentes países europeos. Índice de Aislamiento, para P10, Q1, Q4 y P90 como grupos minoritarios, y promedio

\begin{tabular}{|c|c|c|c|c|c|}
\hline & P10 & Q1 & Q4 & P90 & PROMEDIO \\
\hline ESPAÑA & 0,2091 & 0,3892 & 0,4459 & 0,2426 & 0,3217 \\
\hline ALEMANIA & 0,2264 & 0,3811 & 0,3930 & 0,2266 & 0,3068 \\
\hline AUSTRIA & 0,2489 & 0,3748 & 0,4220 & 0,2454 & 0,3228 \\
\hline BÉLGICA & 0,2283 & 0,3898 & 0,3916 & 0,2118 & 0,3054 \\
\hline BULGARIA & 0,2793 & 0,4180 & 0,4031 & 0,2083 & 0,3272 \\
\hline CROACIA & 0,1786 & 0,3304 & 0,3937 & 0,2409 & 0,2859 \\
\hline DINAMARCA & 0,2001 & 0,3547 & 0,3542 & 0,1972 & 0,2766 \\
\hline ESLOVAQUIA & 0,3683 & 0,4139 & 0,4059 & 0,2375 & 0,3564 \\
\hline ESLOVENIA & 0,2345 & 0,3908 & 0,4112 & 0,2387 & 0,3188 \\
\hline ESTONIA & 0,2146 & 0,3884 & 0,3789 & 0,2314 & 0,3033 \\
\hline FINLANDIA & 0,1584 & 0,3257 & 0,3437 & 0,1713 & 0,2498 \\
\hline FRANCIA & 0,2217 & 0,3862 & 0,4194 & 0,2096 & 0,3092 \\
\hline GRECIA & 0,2285 & 0,3828 & 0,4048 & 0,2466 & 0,3157 \\
\hline HUNGRÍA & 0,3676 & 0,4666 & 0,4718 & 0,2858 & 0,3980 \\
\hline IRLANDA & 0,1713 & 0,3388 & 0,3611 & 0,2012 & 0,2681 \\
\hline ITALIA & 0,2310 & 0,3847 & 0,3865 & 0,2455 & 0,3119 \\
\hline LETONIA & 0,2583 & 0,3893 & 0,3879 & 0,2252 & 0,3152 \\
\hline LITUANIA & 0,2452 & 0,4031 & 0,3887 & 0,1946 & 0,3079 \\
\hline LUXEMBURGO & 0,1583 & 0,3551 & 0,4220 & 0,2114 & 0,2867 \\
\hline MALTA & 0,1716 & 0,3481 & 0,3715 & 0,1971 & 0,2721 \\
\hline PAÍSES BAJOS & 0,2087 & 0,3579 & 0,3846 & 0,2358 & 0,2967 \\
\hline POLONIA & 0,1796 & 0,3487 & 0,3672 & 0,2199 & 0,2789 \\
\hline PORTUGAL & 0,1958 & 0,3730 & 0,4251 & 0,2469 & 0,3102 \\
\hline REINO UNIDO & 0,1751 & 0,3537 & 0,3933 & 0,2184 & 0,2851 \\
\hline REP. CHECA & 0,2388 & 0,3964 & 0,4336 & 0,2689 & 0,3344 \\
\hline RUMANÍA & 0,2549 & 0,4057 & 0,4510 & 0,3204 & 0,3580 \\
\hline SUECIA & 0,1750 & 0,3286 & 0,3560 & 0,1839 & 0,2609 \\
\hline Promedio Unión Europea & 0,2223 & 0,3757 & 0,3975 & 0,2279 & 0,3058 \\
\hline
\end{tabular}

Fuente: elaboración propia con datos de PISA-2015.

Los resultados anteriores muestran que en España la segregación para los estudiantes con familias de mayor nivel socioeconómico es mayor que para los de menor nivel. Este hecho, aunque es el más frecuente en Europa, está lleno de matices. Algunos de ellos son:

1. En 15 de los 27 países analizados la segregación es mayor con el P90 como grupo minoritario que con el P10. Entre los que ocurre lo contrario están algunos países del Este como Hungría y Bulgaria, pero también otros más cercanos al contexto español como Francia, Bélgica, Italia o 
Dinamarca. Para los Qs, solo son seis los países con mayor segregación para los estudiantes con familias de mayor nivel socioeconómico que los de menor.

2. España es el octavo país con mayor diferencia entre el P90 y el P10, con cifras parecidas a Portugal, pero menores que Reino Unido. Sin embargo, es el cuarto país con más diferencia entre Q4 y Q1.

3. Cuanto mayor es la diferencia entre la segregación entre el P10 y el P90 (independientemente del sentido de la diferencia) la segregación promedio aumenta, con una correlación de 0,50 $(\mathrm{p}=, 000)$. Este hecho, sin embargo, no se da en las diferencias entre Q1 y Q4.

\subsection{Segregación escolar en las Comunidades Autónomas}

Vista la panorámica general de España y su comparación con los países de su entorno, el análisis por Comunidades Autónomas amplía la mirada ofreciendo datos muy relevantes. Un primer aspecto que resulta relevante se refiere a que las variaciones entre regiones son muy amplias, tantas como diferencias hay entre países de Europa. Efectivamente, dentro del Estado español encontramos Comunidades como Illes Balears, Galicia y Castilla y León, con índices muy bajos de segregación promedio (comparables a los países nórdicos); y la Comunidad de Madrid, con una segregación más alta que ningún país europeo, con la excepción de Hungría.

De forma análoga a lo presentado anteriormente, en el gráfico 3 se muestran los índices de Gorard (segregación en su dimensión de uniformidad) y de Aislamiento (dimensión de exposición). Allí se encuentran tres grupos de comunidades según su segregación promedio:

1. Comunidades con una segregación escolar por nivel socioeconómico baja (menor o igual a 0,30 según el índice de Gorard y a 0,27 según el de Aislamiento): Illes Balears (0,27 y 0,25), La Rioja $(0,28$ y 0,26), Galicia $(0,29$ y 0,27), Aragón $(0,30,0,26)$, Castilla y León $(0,30$ y 0,26), Cantabria $(0,30$ y 0,27$)$ y Extremadura $(0,30$ y 0,27$)$.

2. Comunidades con una segregación media (entre 0,31 y 0,34 según el índice de Gorard, y entre 0,28 y 0,30 según el de Aislamiento): Comunitat Valenciana (0,31 y 0,28), Región de Murcia (0,31 y 0,28$)$, Andalucía $(0,32$ y 0,29), Comunidad Foral de Navarra (0,33 y 0,28), País Vasco (0,33 y $0,29)$ y Castilla-La Mancha $(0,34$ y 0,30).

3. Comunidades con segregación escolar por nivel socioeconómico alta (más de 0,35 en el índice de Gorard y más de 0,30 en el de Aislamiento): Principado de Asturias (0,35 y 0,31), Cataluña $(0,36$ y 0,31$)$, Canarias $(0,36$ y 0,32$)$ y Comunidad de Madrid $(0,41$ y 0,33$)$. 


\section{Gráfico 3. Segregación escolar por nivel socioeconómico promedio en cada Comunidad Autónoma española. Indices promedio de Gorard y Aislamiento}

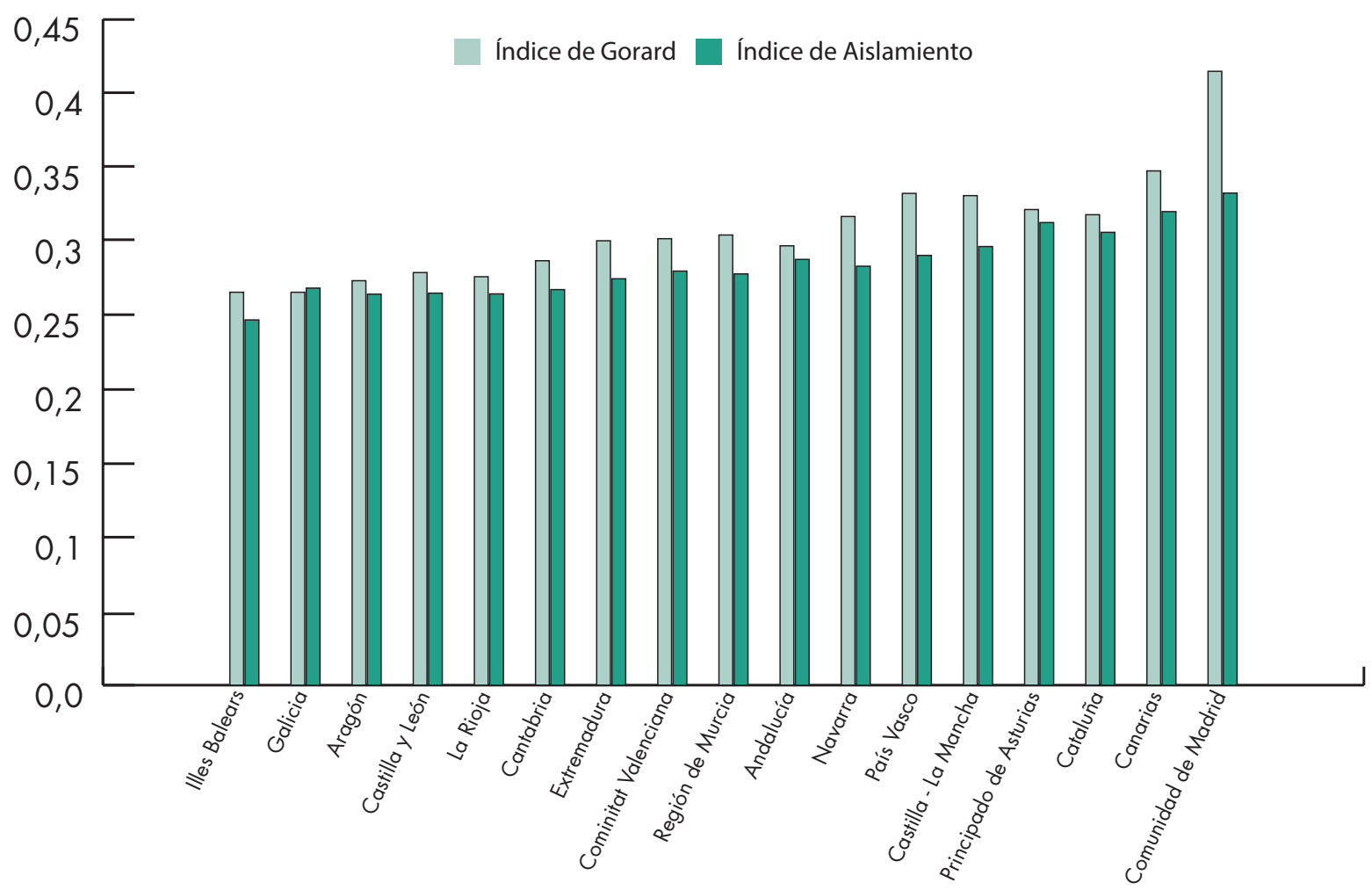

Fuente: elaboración propia a partir de datos de PISA-2015.

Es posible hacer un análisis más detallado en función de los diferentes grupos minoritarios. Así, el orden de las Comunidades varía en función del colectivo estudiado:

- Para el 10\% de estudiantes con menos nivel socioeconómico (P10) las comunidades más segregadas son Comunidad de Madrid $(0,45)$, Navarra $(0,39)$ y el País Vasco $(0,39)$, y las menos Galicia $(0,28)$, Castilla y León $(0,29)$ y Extremadura $(0,30)$.

- Para el 25\% con menos: las que más son la Comunidad de Madrid $(0,36)$, Cataluña $(0,30)$ y La Rioja y el País Vasco $(0,27)$, y las que menos Illes Balears $(0,20)$, Aragón, Cantabria y la Comunitat Valenciana (las tres con 0,22).

- Para el 25\% con mayor nivel socioeconómico: las que más son Comunidad de Madrid $(0,39)$ y Cataluña $(0,35)$, Illes Balears $(0,24)$ y La Rioja $(0,25)$ las que menos.

- Para el 10\% de estudiantes con más nivel socioeconómico, la situación es muy diferente, las que más son Canarias $(0,50)$, Asturias $(0,46)$ y la Comunidad de Madrid $(0,45)$; y las que menos Illes Balears $(0,33)$, La Rioja $(0,35)$ y Aragón y Murcia (ambas con 0,37).

Otros resultados interesantes apuntan a que, a pesar de lo dicho anteriormente, hay tres Comunidades en las que la segregación hacia los estudiantes cuyas familias cuentan con menores recursos es superior a la de mayores, son Navarra y Madrid, con una mínima diferencia entre P10 y P90, y La Rioja, donde la segregación para el Q1 es superior al Q4. En ese ranking, las Comunidades con mayor diferencia de segregación son Canarias, Asturias y Andalucía, y en las que menos diferencia hay son País Vasco y La Rioja. Curiosamente entre las Comunidades Autónomas no se da el fenómeno encon- 
trado entre los países de Europa que se refiere a que una mayor diferencia entre la segregación entre P10 y P90 está relacionado con la segregación promedio, en este caso la correlación es no significativa (tabla 4).

\section{Tabla IV. Segregación escolar por nivel socioeconómico en las Comunidades Autónomas de España. Índice de Gorard para P10, Q1, Q4 y P90 como grupos minoritarios, y promedio}

\begin{tabular}{llllll} 
& P10 & Q1 & Q4 & P90 & PROMEDIO \\
\hline Andalucía & 0,3192 & 0,2606 & 0,2885 & 0,4240 & 0,3231 \\
\hline Aragón & 0,3341 & 0,2221 & 0,2569 & 0,3702 & 0,2958 \\
\hline Principado de Asturias & 0,3414 & 0,2636 & 0,3392 & 0,4575 & 0,3504 \\
\hline Illes Balears & 0,3102 & 0,2019 & 0,2396 & 0,3277 & 0,2699 \\
\hline Canarias & 0,3349 & 0,2569 & 0,3447 & 0,4959 & 0,3581 \\
\hline Cantabria & 0,3073 & 0,2230 & 0,2775 & 0,4063 & 0,3035 \\
\hline Castilla y León & 0,2913 & 0,2274 & 0,2831 & 0,3837 & 0,2964 \\
\hline Castilla - La Mancha & 0,3301 & 0,2623 & 0,3443 & 0,4322 & 0,3422 \\
\hline Cataluña & 0,3693 & 0,3047 & 0,3466 & 0,4145 & 0,3588 \\
\hline Extremadura & 0,3048 & 0,2254 & 0,2689 & 0,3894 & 0,2971 \\
\hline Galicia & 0,2840 & 0,2294 & 0,2647 & 0,3857 & 0,2910 \\
\hline La Rioja & 0,3398 & 0,2712 & 0,2505 & 0,3504 & 0,3030 \\
\hline Comunidad de Madrid & 0,4517 & 0,3632 & 0,3925 & 0,4466 & 0,4135 \\
\hline Región de Murcia & 0,3443 & 0,2438 & 0,2843 & 0,3727 & 0,3113 \\
\hline Com. Foral de Navarra & 0,3917 & 0,2525 & 0,2931 & 0,3800 & 0,3293 \\
\hline País Vasco & 0,3879 & 0,2678 & 0,2794 & 0,3991 & 0,3336 \\
\hline Comunitat Valenciana & 0,3521 & 0,2234 & 0,2793 & 0,3776 & 0,3081 \\
\hline ESPAÑA & 0,4145 & 0,3113 & 0,3542 & 0,4582 & 0,3846 \\
\hline Fina & & &
\end{tabular}

Fuente: elaboración propia con datos de PISA-2015.

Los resultados de la segregación escolar en su dimensión de exposición son similares a los encontrados con la dimensión de uniformidad (tabla 5). No en vano, la correlación entre los índices para cada uno de los grupos minoritarios es de 0,90 para P10, de 0,96 para Q1, de 0,96 para Q4 y de 0,92 para P10. La única diferencia, como se ha señalado, es que este índice está muy influido por el tamaño del grupo minoritario.

Tabla V. Segregación escolar por nivel socioeconómico en las Comunidades Autónomas de España. Índice de Aislamiento para P10, Q1, Q4 y P90 como grupos minoritarios, y promedio

\begin{tabular}{llllll} 
& P10 & Q1 & Q4 & P90 & PROMEDIO \\
\hline Andalucía & 0,1679 & 0,3483 & 0,3961 & 0,2383 & 0,2877 \\
\hline Aragón & 0,1734 & 0,3257 & 0,3628 & 0,1947 & 0,2642 \\
\hline Principado de Asturias & 0,1758 & 0,3504 & 0,4478 & 0,2762 & 0,3126 \\
\hline Illes Balears & 0,1551 & 0,3143 & 0,3407 & 0,1769 & 0,2468 \\
\hline Canarias & 0,1696 & 0,3448 & 0,4633 & 0,3023 & 0,3200 \\
\hline
\end{tabular}


Magnitud de la Segregación escolar por nivel socioeconómico en España y sus Comunidades Autónomas y comparación con los países de la Unión Europea

\begin{tabular}{|c|c|c|c|c|c|}
\hline Cantabria & 0,1562 & 0,3197 & 0,3739 & 0,2192 & 0,2673 \\
\hline Castilla y León & 0,1509 & 0,3222 & 0,3709 & 0,2154 & 0,2649 \\
\hline Castilla - La Mancha & 0,1669 & 0,3463 & 0,4381 & 0,2342 & 0,2964 \\
\hline Cataluña & 0,1721 & 0,3684 & 0,4498 & 0,2337 & 0,3060 \\
\hline Extremadura & 0,1727 & 0,3305 & 0,3761 & 0,2192 & 0,2746 \\
\hline Galicia & 0,1515 & 0,3389 & 0,3802 & 0,2027 & 0,2683 \\
\hline La Rioja & 0,1738 & 0,3501 & 0,3439 & 0,1897 & 0,2644 \\
\hline Comunidad de Madrid & 0,2164 & 0,4270 & 0,4563 & 0,2303 & 0,3325 \\
\hline Región de Murcia & 0,1682 & 0,3343 & 0,3824 & 0,2268 & 0,2779 \\
\hline Com. Foral de Navarra & 0,2009 & 0,3516 & 0,3858 & 0,1941 & 0,2831 \\
\hline País Vasco & 0,2113 & 0,3702 & 0,3746 & 0,2055 & 0,2904 \\
\hline Comunitat Valenciana & 0,1977 & 0,3329 & 0,3838 & 0,2046 & 0,2798 \\
\hline ESPAÑA & 0,2091 & 0,3892 & 0,4459 & 0,2426 & 0,3217 \\
\hline
\end{tabular}

Fuente: elaboración propia con datos de PISA-2015.

\section{Discusión y Conclusiones}

Este estudio ha estimado, con datos actuales, la magnitud de la segregación escolar por nivel socioeconómico y cultural en España, tanto del Estado en su conjunto como de las Comunidades Autónomas; y, además, ha comparado estos resultados con los de países de la Unión Europea. Así, se ha encontrado que la magnitud de la segregación escolar medida a través del índice de Gorard es de 0,38 (segregación en su dimensión de uniformidad), y de 0,32 medido por el índice de Aislamiento (segregación en la dimensión de exposición). Ello significa, por una parte, que el $38 \%$ de los estudiantes debería cambiarse de escuela para lograr un reparto igual a la presencia de los estudiantes de su grupo en la población total. Por otra, que la probabilidad de que un estudiante se encuentre en su escuela con otro estudiante de su mismo grupo es del $32 \%$. Estas cifran, sin embargo, cobran toda su magnitud cuando se enfocan desde dos perspectivas. La primera, se ha encontrado que España es uno de los países con el sistema educativo más segregado de toda la Unión Europea, y muy alejado de los países de su entorno más cercano. Además, si se estima de forma independiente la segregación escolar en cada una de las 17 Comunidades Autónomas, se encuentran magnitudes de la segregación muy dispares.

Esa gran variabilidad en la segregación escolar entre las diferentes Comunidades se muestra con claridad cuando se compara con los países de la Unión Europea (gráfico 4). Así se encuentran, por una parte, Comunidades como Illes Balears o Galicia con una muy baja segregación, justo entre Suecia y Finlandia, los países con menor segregación de Europa y, con ello, del mundo; y, por otra, a la Comunidad de Madrid, con una segregación altísima, entre Hungría y Rumania, los dos países con más segregación de la Unión Europea. 


\section{Gráfico 4. Segregación por nivel socioeconómico promedio en las Comunidades Autónomas y su comparación con diferentes países europeos. Índice de Gorard}

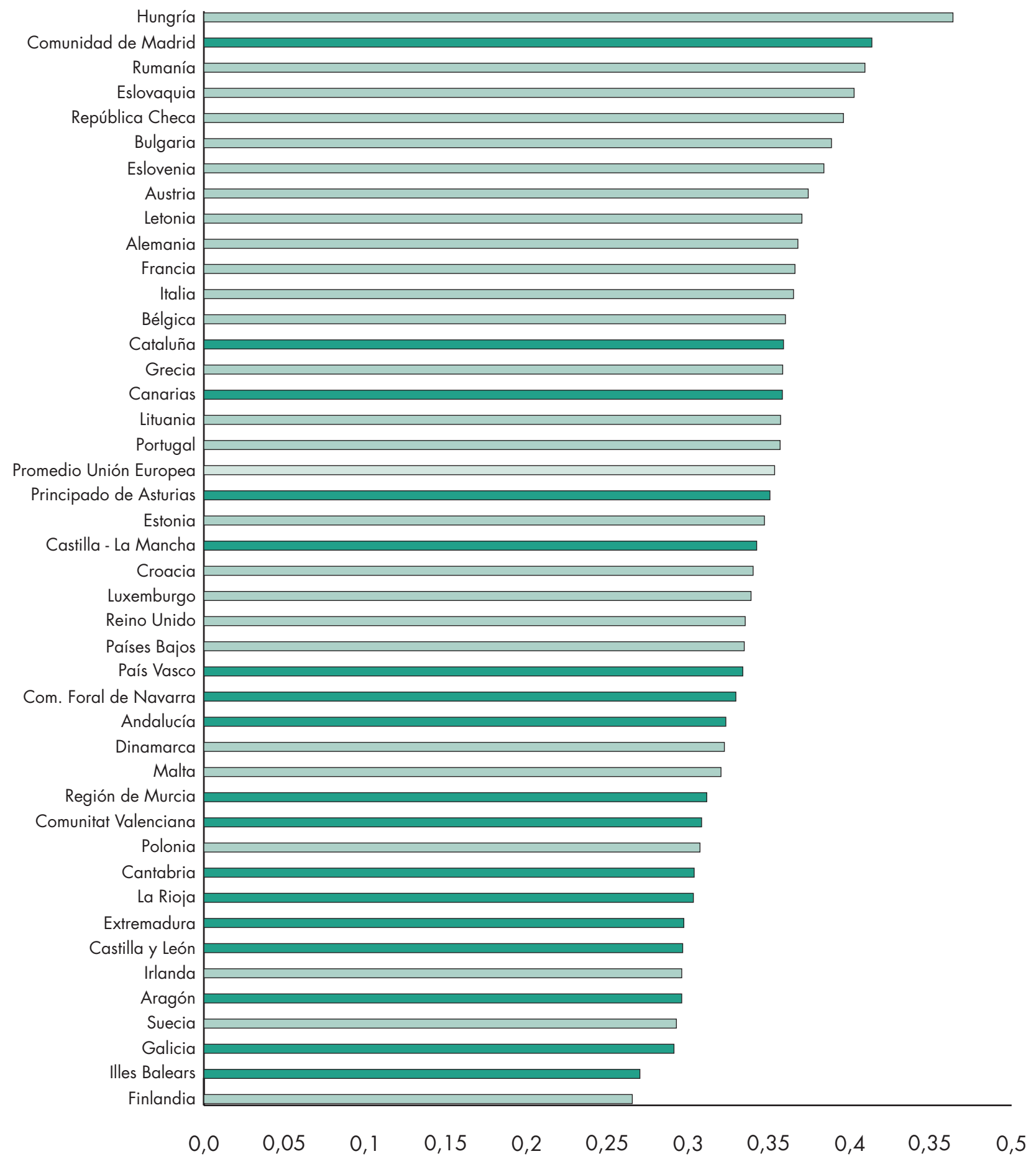

Fuente: elaboración propia a partir de datos de PISA-2015.

Estos resultados son coherentes a los encontrados en otros trabajos análogos anteriores. Los datos globales para España que se han estimado se corresponden a PISA-2006 (Ferrer et al., 2008; MancebónTorrubia y Pérez-Ximénez, 2010) y PISA-2009 (Vázquez, 2012) y, en ambos casos, los autores evidencian que España se encuentra en torno a los 0,39-0,40 puntos (datos estimados mediante el índice de Disimilitud que tal y como expone Murillo (2016) ofrecen puntuaciones algo más altas que el índice de Gorard). Sin embargo, esos datos le hacen estar en una situación promedio entre los países de su contexto. No 
sería de extrañar que la crisis económica hubiera hecho que la segregación escolar en España hubiera crecido de forma importante en estos años. Se deja como una hipótesis a validar en el futuro.

Aunque hasta el momento no se había realizado ningún estudio sobre segregación escolar en las diferentes Comunidades Autónomas, las grandes variaciones encontradas tienen su correlato a lo hallado entre regiones de otros países. Como antes se señaló, Gorard y Hordsoy (2013), por ejemplo, encontraron 0,16 puntos de diferencia entre regiones; de acuerdo con Dupriez y Vandenberghe (2004) estas diferencias son de hasta 8 décimas en Bélgica. En España las diferencias alcanzan los 1,3 puntos, por lo que entran dentro de lo razonable a pesar de lo impactante.

En este estudio se ha trabajado con datos de PISA-2015. Cierto es que se cuenta con pocos estudiantes por escuela, lo que nos hace estar alerta acerca de la fiabilidad de este estudio, pero el tamaño de la muestra, la estrategia de muestreo y la seriedad con la que se recogen los datos hacen que sea una herramienta extremadamente útil para este propósito. Que todas las Comunidades hayan ampliado su muestra en la prueba de 2015 frente a años anteriores es una oportunidad para conocer más y mejor sistema educativo en España. Ojalá que las Administraciones educativas pongan a disposición de la comunidad científica los datos de las múltiples evaluaciones que se están realizando en la actualidad. Será una excelente forma de profundizar en el conocimiento de la calidad y la equidad de las escuelas. Es importante destacar en estas conclusiones el desarrollo metodológico realizado contribuyendo al uso de índices de segregación alternativos, no derivados de los estudios de segregación residencial y más adecuados a la realidad escolar que se analiza; por ejemplo, a través de Modelos Multinivel. Goldstein y Noden (2003) y Leckie y colaboradores (2012) están trabajando en la incorporación del uso de estas técnicas avanzadas para el cálculo de la segregación escolar, pero se han centrado en variables dicotómicas.

Este trabajo, más que respuestas, genera un buen número de interrogantes. Conocida la magnitud de la segregación escolar por nivel socioeconómico hay que preguntarse por las razones de la misma y sus consecuencias. Interesa averiguar, también, cómo evolucionan estos datos, tanto para analizar el impacto de las políticas realizadas, como el generado por la crisis económica. Y hay que seguir profundizando en metodologías más adecuadas para medir la segregación. Al final lo importante es que la segregación escolar sea un tema preferente de estudio que genere una preocupación por parte de administradores, políticos y de la sociedad en general, acerca de su magnitud y consecuencias.

Sin duda alguna, uno de los retos más importantes que tiene la educación española en la actualidad es el de la equidad, por encima incluso del desafío de la calidad. Sin embargo, aún se conoce poco de la situación de la equidad de las escuelas españolas, especialmente en el estudio del nivel socioeconómico. No cabe duda de que el primer paso para cambiar las injusticias es conocerlas y denunciarlas. La segregación escolar es producto de muchos factores. Uno de ellos es la segregación residencial, pero no solo. Las políticas educativas favorecen o limitan la segregación de la escuela. Decisiones tales como el fomento de mecanismos de cuasi-mercado potenciando opciones de elección de centro, por ejemplo, favorecen la segregación escolar (Taylor et al., 2003).

Y la mejor muestra de ello son los resultados encontrados en la presente investigación. La Comunidad de Madrid es, sin duda alguna, la región de España que más ha impulsado la creación de sistemas de cuasimercado escolar, así como políticas de selección escolar. Así, por ejemplo, en los últimos años se ha fomentado decididamente la competencia entre centros y la libre elección por parte de familias y estudiantes. En esta lógica se encuentran medidas tales como el establecimiento de un distrito único, por el cual 
las familias pueden elegir libremente el centro entre todos los de la región, o la publicación de rankings de centros atendiendo a los resultados de pruebas estandarizadas externas en centros de Educación Infantil y Primaria y de Educación Secundaria. Junto a ello, la discutida política de creación de centros bilingües que está generando que procesos de selección implícitos por los cuales los niños, niñas y adolescentes con más recursos asisten a este tipo de centros, dejando a los que más dificultades tienen fuera de los mismos. Tampoco es posible olvidar la apuesta decidida por fomentar la educación privada, siendo la Comunidad de Madrid la región de España con más estudiantes matriculados en centros de titularidad privada.

La segregación escolar es un poderoso mecanismo para impedir la igualdad de oportunidades real entre todos los estudiantes; así como el mejor medio para fomentar la segregación y las desigualdades sociales. Cierto es que una gran parte de la segregación escolar es consecuencia directa de la segregación residencial; mal que afecta a prácticamente todas las ciudades de España. Pero no lo es menos que la segregación escolar es un claro producto de las medidas políticas tomadas al respecto; medidas que pueden favorecer la segregación o impedirla. Los datos de España así lo ratifican. Es hora de empezar a pensar qué sistema educativo queremos, porque en la medida que éste sea más o menos segregado, así será la sociedad que construyamos para el futuro.

\section{Referncias bibliográficas}

Allen, Rebecca y Vignoles, Anna (2007): “What Should an Index of School Segregation Measure?” Oxford Review of Education, 33(5), 643-668. https://doi.org/10.1080/03054980701366306.

Andersson, Roger y Kährik, Anneli (2015): “Widening Gaps: Segregation Dynamics During Two Decades of Econocimic and Institutional Change in Stockholm" en Tiit Tammaru, Szymon Marcińczak, Maarten van Ham y Sako Musterd (Eds.): Socio-economic segregation in European capital cities: East meets west. Nueva York, NY: Routledge.

Benito, Ricard y González-Balletbó, Isaac (2007). Processos de segregació escolar a Catalunya. Barcelona: Mediterrània.

Benito, Ricard; Alegre, Miguel Angel y González-Balletbó, Isaac (2014): "School Segregation and its Effects on Educational Equality and Efficiency in 16 OECD Comprehensive School Systems". Comparative Education Review, 58 (1), 104-134. https://doi.org/10.1086/672011.

Bonal, Xavier (2012). Municipis contra la segregació escolar. Sis experiències de politica educativa local. Barcelona: Fundació Jaume Bofill.

Cheng, Shou Chen y Gorard, Stephen (2010): "Segregation by Poverty in Secondary Schools in England 2005-2009”. Journal of Education Policy, 25 (3), 415-418. https://doi.org/10.1080/02680931003699542.

Coleman, James Samuel; Kelly, Sara y Moore, Jack (1975). Trends in school segregation, 1968-73. Washington, DC: The Urban Institute.

Duncan, Otis y Duncan, Beverly (1955): "A Methodological Analysis of Segregation Indexes". American Sociological Review, 20, 210-217. https://doi.org/10.2307/2088328.

Dupriez, Vincent (2010). Methods of Grouping Learners at School. París: UNESCO.

Dupriez, Vincent y Vandenberghe, Vincent (2004): "L'école en communauté française de Belgique: De quelle inégalité parlons-nous?” Les Cahiers de Recherche en Éducation et Formation, 27, 3-26. 
Echenique, Federico y Fryer, Roland (2007): "A Measure of Segregation Based on Social Interactions". The Quarterly Journal of Economics, CXXII(2), 441-485. https://doi.org/10.1162/qjec.122.2.441.

Fernández-Enguita, Mariano (2008): "Escuela pública y privada en España: La segregación rampante". RASE: Revista de la Asociación de Sociología de la Educación, 1 (2), 42-69.

Ferrer, Ferrán; Ferrer, Gloria y Castel, José Luis (2008). Les desigualtats educatives a Catalunya (I-II). Barcelona: Fundació Jaume Bofill.

Ferrer, Ferrán; Castejón, Alba; Castel, José Luis y Zancajo, Adriá (2011). PIS A 2009: Evaluació de les desigualtats educatives a Catalunya. Barcelona: Fundació Jaume Bofill.

Frankenberg, Erica; Lee, Chungmei y Orfield, Gary (2003). A Multiracial Society with Segregated Schools: Are we Losing the Dream? Boston, MA: Harvard University.

Frankel, David y Volij, Oscar (2011): “Measuring School Segregation”. Journal of Economic Theory, 146 (1), 1-38. https://doi.org/10.1016/j.jet.2010.10.008.

Goldstein, Harvey y Noden, Philip (2003): "Modelling Social Segregation”. Oxford Review of Education, 29 (2), 225-237. https://doi.org/10.1080/0305498032000080693.

Goodman, Frank (1972): “De Facto School Segregation: A Constitutional and Empirical Analysis”. California Law Review, 60 (2), 275-437. https://doi.org/10.2307/3479538.

Gorard, Stephen (1998): “The Missing Impact of Marketization”. School Leadership and Management, 17 (3), 437-438.

Gorard, Stephen (2006). What does an Index of School Segregation Measure? A Commentary on Allen and Vignoles. York: Department of Educational Studies.

Gorard, Stephen y Hordsoy, Rita (2013): "Narrowing Down the Determinants of Between-school Segregation: An Analysis of the Intake to all Schools in England, 1989-2011". Journal of School Choice, 7(2), 182-195. https://doi.org/10.1080/15582159.2013.791182.

Gorard, Stephen y Taylor, Chris (2002): "What is Segregation? A Comparison of Measures in Terms of 'Strong' and 'Weak' Compositional Invariance”. Sociology, 36 (4), 875-895. https://doi. org/10.1177/003803850203600405.

Hutchens, Robert (2004): “One Measure of Segregation”. International Economic Review, 45 (2), 555-578. https://doi.org/10.1111/j.1468-2354.2004.00136.x.

Ireson, Judith y Hallam, Susan (2001). Ability Grouping in Education. Londres: Paul Chapman Publishing. Jenkins, Stephen; Micklewright, John y Schnepf, Sylke (2008): "Social Segregation in Secondary Schools: How does England Compare with other Countries?”. Oxford Review of Education, 34 (1), 21-37. https:// doi.org/10.1080/03054980701542039.

Johnston, Robert, Wilson, Deborah y Burgess, Simon (2004): "School Segregation in Multiethnic England”. Ethnicities, 4 (2), 237-265. https:/ / doi.org/10.1177/1468796804042605.

Karsten, Sjoerd (2010): "School segregation" en OCDE. (Ed.): Equal opportunities? The labour market integration of the children of inmigrants. París: OCDE. 
Katz, Irwin (1964): "Review of Evidence Relating to Effects of Desegregation on the Intellectual Performance of Negroes". American Psychologist, 19 (6), 381-399. https://doi.org/10.1037/h0040569.

Leckie, Georgey; Pillinger, Rebecca; Jones, Kelvin y Goldstein, Harvey (2012): "Multilevel Modeling of Social Segregation”. Journal of Educational and Behavioral Statistics, 37 (1), 3-30. https://doi. org/10.3102/1076998610394367.

Lieberson, Stanley (1981): “An asymmetrical approach to segregation” en C. Peach (Ed.): Ethnic segregation in cities. Londres: Croom-Helm.

Mancebón-Torrubia, María Jesús y Pérez-Ximénez de Embún, Domingo (2005): "El alumnado de los centros educativos aragoneses de enseñanza secundaria: Segregación socioeconómica y académica por tipo de centro y percepciones de la calidad educativa". Economía Aragonesa, 35, 115-136.

Mancebón-Torrubia, María Jesús y Pérez-Ximénez de Embún, Domingo (2009): "Segregación escolar en el sistema educativo español. Un análisis a partir de PISA 2006". Investigaciones de Economía de la Educación, 4, 63-77.

Mancebón-Torrubia, María Jesús y Pérez-Ximénez de Embún, Domingo (2010): “Una valoración del grado de segregación socioeconómica existente en el sistema educativo español. Un análisis por comunidades autónomas a partir de PISA 2006”. Regional and Sectoral Economic Studies, 10 (3), 129-148.

Murillo, F. Javier (2016): "Midiendo la segregación escolar en América Latina. Un análisis metodológico utilizando el TERCE". REICE. Revista Iberoamericana sobre Calidad, Eficacia y Cambio en Educación, 14 (4), 33-60. https://doi.org/10.15366/reice2016.14.4.002.

Murillo, F. Javier y Martínez-Garrido, Cynthia (2017a): "Estimación de la magnitud de la segregación escolar en América Latina”. Magis, Revista Internacional de Investigación en Educación, 9(19), 11-30. https:/ / doi.org/10.11144/javeriana.m9-19.emse.

Murillo, F. Javier; Martínez-Garrido, Cynthia y Belavi, Guillermina (2017b): “Segregación escolar por origen nacional en España”. OBETS. Revista de Ciencias Sociales, 12 (2), 395-423. https:/ / doi.org/10.14198/ OBETS2017.12.2.04.

OCDE. (2015). PISA 2015 Technical report. Sample design. Recuperado de http://www.oecd.org/pisa/ data/2015-technical-report/.

OCDE. (2016). Programme of International Student Assessment (PIS A 2015). París: OCDE.

Orfield, Gary (2009). Reviving the Goal of an Integrated Society: A 21st Century Challenge. Los Angeles, CA: University of California Press.

Orfield, Gary y Eaton, Susan (1997). Dismantling Desegregation: The Quiet Reversal of Brown v. Board of Education. Nueva York, NY: New Press.

Orfield, Gary; Siegel-Hawley, Genevieve y Kucsera, John (2014). Sorting Out Deepening Confusion on Segregation Trends. Los Angeles, CA: The Civil Rights Project.

Peach, Boal (1975). Urban Residential Segregation. Londres: Longman.

Reardon, Sean y Owens, Ann (2014): "60 years after Brown: Trends and Consequences of School Segregation”. Annual Review of Sociology, 40, 199-218. https://doi.org/10.1146/annurev-soc-071913-043152. 
Sánchez Hugalde, Adriana (2007): "La segregació escolar dels immigrants a Catalunya". Quaderns D'avaluació, 13, 55-76.

Síndic. (2008). La segregación escolar en Cataluña. Barcelona: SINDIC

Stephan, Walter (Ed.). (2013). School Desegregation: Past, Present, and Future. Nueva York, NY: Springer.

Stroub, Kori y Richards, Meredith (2013): "From Resegregation to Reintegration: Trends in the Racial/ Ethnic Segregation of Metropolitan Public Schools, 1993-2009”. American Educational Research Journal, 50 (3), 497-531. https://doi.org/10.3102/0002831213478462.

Taylor, Chris; Gorard, Stephen y Fitz, John (2000): “A Re-examination of Segregation Indices in Terms of Compositional Invariance". Social Research Update, 30, 1-24.

Taylor, Chris; Gorard, Stephen y Fitz, John (2003): “The Modifiable Areal Unit Problem: Segregation Between Schools and Levels of Analysis”. International Journal of Social Research Methodology, 6 (1), 41-60. https://doi.org/10.1080/13645570305053.

Vázquez, Emmanuel (2012). Segregación escolar por nivel socioeconómico. Midiendo el fenómeno y explorando sus determinantes. Buenos Aires: CEDLAS.

Watson, Tara (2009): "Inequality and the Measurement of Residential Segregation by Income in American Neighborhoods". Review of Income and Wealth, 55 (3), 820-844. https://doi.org/10.3386/w14908.

Wilson, William (1987). The Truly Disadvantaged. Chicago, IL: University of Chicago Press.

\section{Notas biográficas}

F. Javier Murillo es profesor titular del Área de Métodos de Investigación y Diagnóstico en Educación de la Universidad Autónoma de Madrid. Coordinador del grupo de investigación «Cambio Educativo para la Justicia Social» y Secretario del Instituto de Derechos Humanos, Democracia y Cultura de Paz y No Violencia (DEMOSPAZ).de la UAM. Fue Coordinador General del Laboratorio Latinoamericano de Evaluación de la Calidad de la Educación (LLECE), de la UNESCO, y Director de Estudios del Centro de Investigación y Documentación Educativa (CIDE) del Ministerio de Educación de España. Página web: www.fjaviermurillo.es.

Cynthia Martínez-Garrido es profesora Ayudante Doctor en Métodos de Investigación y Evaluación en Educación en la Universidad Autónoma de Madrid. Miembro del grupo de investigación «Cambio Educativo para la Justicia Social». Editora de tres revistas de investigación científica REICE. Revista Iberoamericana sobre Calidad, Eficacia y Cambio en Educación, Revista Internacional de Educación para la Justicia Social y Revista Latinoamericana de Educación Inclusiva. Sus líneas de investigación son: Enseñanza eficaz, Segregación escolar, Liderazgo educativo y Educación para la Justicia Social. Página web: www.cmartinezgarrido.es. 